\title{
A Review of Cardiac Image Registration Methods
}

\author{
Timo Mäkelä*, Student Member, IEEE, Patrick Clarysse, Outi Sipilä, Nicoleta Pauna, Quoc Cuong Pham, \\ Toivo Katila, Member, IEEE, and Isabelle E. Magnin, Member, IEEE
}

\begin{abstract}
In this paper, the current status of cardiac image registration methods is reviewed. The combination of information from multiple cardiac image modalities, such as magnetic resonance imaging, computed tomography, positron emission tomography, single-photon emission computed tomography, and ultrasound, is of increasing interest in the medical community for physiologic understanding and diagnostic purposes. Registration of cardiac images is a more complex problem than brain image registration because the heart is a nonrigid moving organ inside a moving body. Moreover, as compared to the registration of brain images, the heart exhibits much fewer accurate anatomical landmarks. In a clinical context, physicians often mentally integrate image information from different modalities. Automatic registration, based on computer programs, might, however, offer better accuracy and repeatability and save time.
\end{abstract}

Index Terms-Cardiac image registration, computed tomography (CT), magnetic resonance imaging (MRI), positron emission tomography (PET), single-photon emission computed tomography (SPECT), ultrasound (US).

\section{NOMENCLATURE}

$\begin{array}{ll}\text { CC } & \text { Correlation coefficient. } \\ \text { CT } & \text { X-ray computed tomography. } \\ \text { ECG } & \text { Electrocardiography. } \\ \text { ED } & \text { End-diastolic. } \\ \text { ES } & \text { End-systolic. } \\ \text { FDG } & \text { Fluorodeoxyglucose. } \\ \text { ICP } & \text { Iterative closest point. } \\ \text { LA } & \text { Long axis. } \\ \text { LV } & \text { Left ventricle. } \\ \text { MRI } & \text { Magnetic resonance imaging. } \\ \text { PET } & \text { Positron emission tomography. } \\ \text { rms } & \text { Root mean square. } \\ \text { SA } & \text { Short axis. } \\ \text { SAD } & \text { Sum of absolute differences. } \\ \text { SPECT } & \text { Single-photon emission computed tomography. } \\ \text { SSC } & \text { Stochastic sign change. } \\ \text { SSD } & \text { Sum of squared intensity differences. }\end{array}$

Manuscript received November 3, 2001; revised July 6, 2002. This work was supported in part by the Scientific Department of the French Embassy in Finland, the Region Rhône Alpes, the ADéMO project, and The Foundation of Technology in Finland. Asterisk indicates corresponding author.

*T. Mäkelä is with the Laboratory of Biomedical Engineering, Helsinki University of Technology, P.O. Box 2200, FIN-02015 HUT, Finland, and CREATIS, UMR CNRS \#5515, 69621 Villeurbanne Cedex, France (e-mail: timo.makela@hut.fi).

P. Clarysse, N. Pauna, Q. C. Pham, and I. E. Magnin are with CREATIS, UMR CNRS \#5515, 69621 Villeurbanne Cedex, France.

O. Sipilä is with the Department of Radiology, Helsinki University Central Hospital, FIN-00029 HUS, Finland.

T. Katila is with the Laboratory of Biomedical Engineering, Helsinki University of Technology, FIN-02015 HUT, Finland, and BioMag Laboratory, Helsinki University Central Hospital, FIN-00029 HUS, Finland

Digital Object Identifier 10.1109/TMI.2002.804441

\author{
SVD Singular value decomposition. \\ US Ultrasound. \\ VIR Variance of intensity ratio.
}

\section{INTRODUCTION}

D IFFERENT imaging modalities bring complementary information that can be advantageously used to establish a diagnosis or assist the clinician for a therapeutic gesture. To locally compare two or more measurements of different nature, a number of registration algorithms have been developed, especially in brain imaging.

In the widespread ischemic heart diseases, the consequence of reduced blood flow to the heart muscle can be studied using several medical imaging modalities, each of which gives a specific view of this complex phenomenon. The first consequence is a deterioration of the myocardial perfusion, which can be analyzed with nuclear medicine imaging techniques (SPECT and PET) or with MRI [1], [2]. The deficit of perfusion induces metabolic changes in myocardial tissues highlighted using FDG PET studies [1]. A further consequence of a myocardial ischemia is the reduced capacity of the heart to eject blood into the body. This can be evaluated by analyzing the myocardial contractile function using MRI or US. Recent studies have demonstrated the interest of concurrently analyzing those different aspects in order to assess the myocardial viability, which will determine the proper therapeutic action [3], [4]. Therefore, there is a growing interest in the development of cardiac image registration methods that could bring into the same anatomical reference all the available functional measurements.

Cardiac image registration is a more complex problem than brain image registration, in particular because of the nonrigid and mixed motions of the heart and the thorax structures. Moreover, as compared to the brain, the heart exhibits fewer accurate anatomical landmarks. Also, cardiac images are usually acquired with a lower resolution than brain images. Fig. 1 illustrates a typical acquisition protocol with ECG-gated cardiac MRI. This highlights certain problems induced by the spatiotemporal image acquisition that hamper image registration.

In clinical practice, physicians mentally integrate information from different images acquired from a patient, often with different imaging modalities. Images are shown in various orientations and positions and at different scales. Semi-interactive registration methods rely on the expert's ability to interactively select corresponding slices using anatomical knowledge. In cardiac image registration, the semi-interactive methods are often used to register gated SA images [5], [6].

Several survey papers have been published in the field of medical image registration [7]-[10]. Also, some review articles 


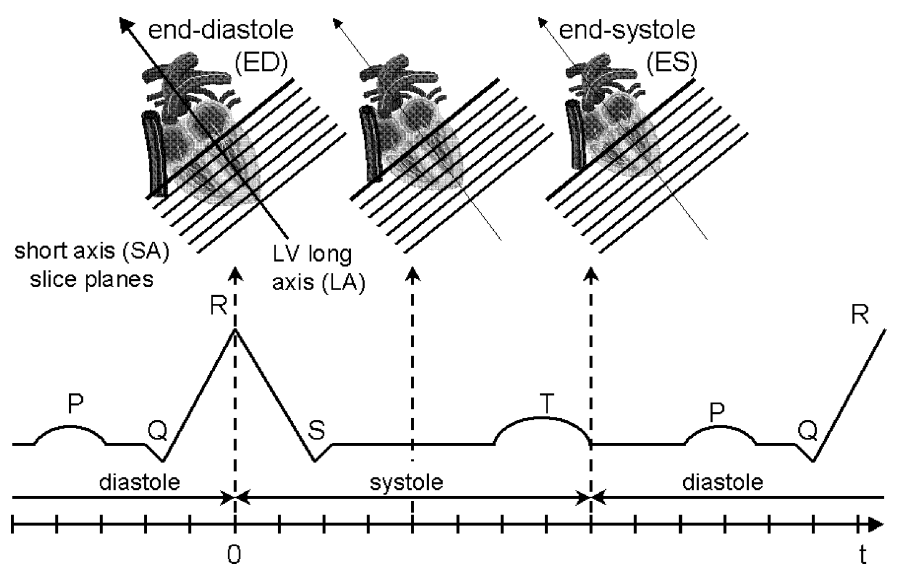

Fig. 1. Illustration of a classical acquisition of SA images with a ECG-gated cine MR sequence. The same slice is acquired at successive time points of the cardiac cycle. However, due to the motion of the heart, we do not observe the same anatomical region within the same slice. Moreover, several cardiac cycles are required to reconstruct slices. When possible, patients are asked to retain their breath (15-20 s) during acquisition.

and books closely related to medical image registration and fusion can be mentioned, such as [11]-[17]. Very few review papers focusing on cardiac image registration have been published [18], [19]. Gilardi et al. [18] reviewed the techniques and clinical applications for the integration of multimodal biomedical images of the heart. Habboosh et al. [19] briefly discussed the aspects of cardiac PET and MRI correlation. In the review article of Maintz et al. [8], registration methods for cardiac images were also referenced in a separate section.

This paper aims to provide a survey concerning cardiac image registration, including the most recent articles and discussing also implementation and validation issues. For the four-dimensional (4-D) registration of intramodality cardiac images, the problem is often addressed in a cardiac motion tracking framework. In this survey, the 4-D motion tracking problem is not considered at the methodological level. Instead, we refer to some recent papers in this field [14], [20]-[23].

In this paper, cardiac image registration methods are divided into two main categories: 1) those based on geometric image features (Section II-A) and 2) those based on voxel similarity measures (Section II-B). The geometric image feature-based methods are divided into registration of a set of points and edges or surfaces. Registration methods based on voxel similarity measures include moments and principal-axes methods, intensity difference and correlation methods, and methods based on mutual information. After presenting the current status of cardiac image registration methods, we discuss implementation issues, such as interpolation and optimization (Section II-C). Validation of the registration methods is presented in Section III. The overview of cardiac and thorax registration methods and their main parameters are summarized in Table I. Some of the brain, thorax, and abdominal image registration articles are referenced when methods have been, or can easily be, applied to heart image registration.

In the reviewed papers, mainly MR, CT, PET, and SPECT imaging modalities have been considered. There are very few references related to registration with cardiac US images. This is due to the characteristics of the US images that make them diffi- cult to process automatically. Moreover, despite the existence of three-dimensional (3-D) echocardiographic systems [24], twodimensional (2-D) image acquisitions are routinely performed. At most, a collection of radial planes is acquired, resulting in a quite different geometry as compared to the other imaging modalities.

\section{CARdiac Image Registration: PRINCIPLES AND APPLICATIONS}

\section{A. Registration Methods Based on Geometric Image Features}

Registration methods based on geometric image features can be divided into registration of a set of points and registration of edges or surfaces.

1) Point-Based Registration: Point-based registration methods often uses external markers or anatomical landmarks. Corresponding point sets are usually manually defined in the reference and floating images. The advantages of the pointbased registration methods are that they can be applied to any imaging modalities where markers or landmarks are visible and that the calculation of the registration parameters between two point sets is usually fast. A noniterative least squares method can be used to register corresponding point sets [12], [25]. The method uses an SVD of a 3 by 3 covariance matrix to find a unique solution for the registration parameters between two point sets. In cardiac image registration, the method has been used, e.g., in phantom experiments for validating rigid registration error [26].

Registration methods based on external skin markers (fiducial markers) are widely applied in medical image registration because they allow matching of any imaging modalities in which the positions of markers can be accurately defined. Registration based on skin markers is independent of the alteration in the image patterns, induced by the pathologies [18]. Skin markers must be easy to use and accurate to reattach, but they should not interfere with the diagnostic content of the images. Since the flexibility of the body can cause errors in registration, it is important to choose relatively stable parts of the body for markers placement [27]. Ideally, markers should not be removed between imaging sessions to ensure the same placements in different modalities [28]. Registration of external skin markers does not guarantee registration of the heart within the body, since heart position changes with body position, respiration, and cardiac contraction [29]. The disadvantage of the skin markers is also that they cannot be utilized retrospectively. External markers have been applied especially as a gold standard method in phantom measurements to validate the accuracy of rigid cardiac and thorax registration methods [26], [30]-[33] and in clinical images, e.g., to validate the accuracy of rigid registration of head images [34]. For rigid thorax CT and SPECT image registration, the combination of both external markers and landmarks has also been utilized [35], [36].

In landmark-based registration, corresponding anatomical points have to be visible in both registered images. For heart images, there are usually only few spatially accurate anatomical landmarks. In pathological conditions, such as ischemia, the functional alterations can also hide anatomical landmarks [18]. 
TABLE I

Overview of Evaluated CardiaC and Thorax IMage Registration Methods

\begin{tabular}{|c|c|c|c|c|c|c|c|c|}
\hline Reference & Modalities & Object & Trans. & Struc. & Method & Valid. & Error & Error type \\
\hline \multicolumn{9}{|c|}{ Registration methods based on geometric image features } \\
\hline \multicolumn{9}{|l|}{ Point-based registration } \\
\hline Wirth et al. [42] & CT-MR & Thorax & Elastic & Landmarks & $\begin{array}{l}\text { Interpolation \& } \\
\text { Elastic funct. }\end{array}$ & - & - & - \\
\hline \multicolumn{9}{|c|}{ Thorax surface based registration } \\
\hline Yu et al. [33] & CT-PET & Thorax & Rigid & T\&L & "head-and-hat" & $\overline{\mathrm{P}}$ & $\begin{array}{llll}(\mathrm{x}, \mathrm{y}) & 2.3 \mathrm{~mm}, & (\mathrm{y}) \\
3.0 \mathrm{~mm} & & \end{array}$ & mean $(\mathrm{rms})$ \\
\hline Cai et al. [46] & CT-PET & Lungs & Rigid & $T \& L$ & Chamfer & $\begin{array}{l}\text { P\& } \\
\text { Pa.\& S }\end{array}$ & $\begin{array}{l}\text { (x,y) } 2-3 \mathrm{~mm},(\mathrm{y}) \\
3-4 \mathrm{~mm},(\text { rot.) }) 1.5^{\circ}\end{array}$ & mean \\
\hline Pallotta et al. [26] & PET-PET & Heart & Rigid & $\mathrm{T} \& \mathrm{~L}$ & Chamfer & $P \& S$ & $\begin{array}{l}3 \mathrm{~mm} \text {, (rot.) } 1^{\circ} \\
2.19 \pm 0.52 \mathrm{~mm}\end{array}$ & $\begin{array}{l}\text { mean }(\mathrm{rms}) \\
\text { mean }(\mathrm{rms}) \pm \text { s.d. }\end{array}$ \\
\hline Gilardi et al. [31] & SPECT-PET & Heart & Rigid & $\mathrm{T} \& \mathrm{~L}$ & Chamfer & $\mathrm{P} \& \mathrm{~Pa}$ & $(\mathrm{x}, \mathrm{y}) \overline{3 \mathrm{~mm}}, 5 \mathrm{~mm}(\mathrm{z})$ & $\begin{array}{l}(\mathrm{x}, \mathrm{y}) \text { mean }(\mathrm{rms}) \\
(\mathrm{z}) \text { mean }\end{array}$ \\
\hline Mäkelä et al. [47] & MR-PET & Heart & Rigid & $\mathrm{T} \& \mathrm{~L}$ & Chamfer & Surfaces & $(\mathrm{x}, \mathrm{y}, \mathrm{z}) 2.8 \pm 0.5 \mathrm{~mm}$ & mean \\
\hline \multicolumn{9}{|c|}{ Heart surface based registration } \\
\hline Faber et al. [29] & MR-SPECT & Heart & Rigid & HS & "head-and-hat" & $\mathbf{P}$ & $2.7 \mathrm{~mm}$ & mean (rms) \\
\hline Sinha et al. [40] & MR-PET & Heart & Rigid & HS & "head-and-hat" & L & $1.95 \mathrm{~mm} \pm 1.6 \mathrm{~mm}$ & mean (rms) \\
\hline Nekolla et al. [57] & PET-SPECT & Heart & Rigid & HS & - & Surfaces & $2.5 \mathrm{~mm}$ & mean \\
\hline Declerck et al. [50] & SPECT-SPECT & Heart & Elastic & HS & ICP & $\mathrm{Pa}$. & - & - \\
\hline \multicolumn{9}{|c|}{ Registration methods based on voxel similarity measures } \\
\hline \multicolumn{9}{|c|}{ Intensity difference and correlation methods } \\
\hline Gallippi et al. [75] & $\begin{array}{l}\text { MR-MR } \\
\text { (time series) }\end{array}$ & Heart & $\begin{array}{l}\text { Rigid \& } \\
\text { Elastic }\end{array}$ & - & $\bar{C}$ & $\overline{\mathbf{M}}$ & $\begin{array}{l}1.23 \pm 0.06 \mathrm{~mm} \\
3.25 \pm 1.04 \mathrm{~mm}\end{array}$ & $\begin{array}{l}\text { left-right (mean) } \\
\text { anterior-poster. } \\
\text { (mean) }\end{array}$ \\
\hline Bidaut et al. [37] & $\begin{array}{l}\text { MR-MR } \\
\text { (perfusion) }\end{array}$ & Heart & Rigid & - & $S S D$ & L & $\begin{array}{l}3.0 \mathrm{~mm}(\mathrm{x}), \\
1.6 \mathrm{~mm}(\mathrm{y}), 2.2 \mathrm{~mm}(\mathrm{z})\end{array}$ & $\begin{array}{l}\text { mean }(\mathrm{rms}) \\
(\text { maximum) }\end{array}$ \\
\hline Bacharach et al. [76] & PET-PET & Heart & Rigid & - & $C C$ & $\mathbf{M}$ & $\begin{array}{l}(\mathrm{x}, \mathrm{y}, \mathrm{z}) \\
1.5^{\circ}\end{array} 1 \mathrm{~mm}, \quad$ (rot.) & mean \\
\hline Turkingston et al. [71] & PET-PET & Heart & Rigid & - & $C$ & $\mathbf{P}$ & $\begin{array}{l}(\mathrm{x}, \mathrm{y}) 1.7 \mathrm{~mm},(\mathrm{z}) 4.2 \\
\mathrm{~mm}\end{array}$ & mean \\
\hline Klein et al. [23] & PET-PET & Heart & $\begin{array}{l}\text { Elastic } \\
\text { (4-D) }\end{array}$ & - & LS & $\mathbf{P}$ & $\begin{array}{l}\text { (x) } 1.9 \mathrm{~mm},(\mathrm{y}) 2.4 \\
\mathrm{~mm},(\mathrm{z}) 6.8 \mathrm{~mm}\end{array}$ & mean (max.) \\
\hline Hoh et al. [67] & MR-SPECT & Heart & Rigid & - & $S A D, S S C$ & M & $\begin{array}{l}\text { (x,y) } 0.5 \pm 0.5 \mathrm{~mm},(\mathrm{z}) \\
1.1 \pm 1.1 \mathrm{~mm},(\mathrm{rot}) 0.9 \\
\pm 1.1 \circ\end{array}$ & mean \pm s.d. \\
\hline Dey et al. [32] & CT-SPECT & $\begin{array}{l}\text { Heart \& } \\
\text { Thorax }\end{array}$ & Rigid & - & $\begin{array}{l}S A D \\
V I R\end{array}$ & $\begin{array}{l}P \\
P\end{array}$ & $\begin{array}{l}2.5 \pm 1.2 \mathrm{~mm} \\
3.3 \pm 1.3 \mathrm{~mm}\end{array}$ & $\begin{array}{l}\text { mean }(\mathrm{rms}) \\
\text { mean }(\mathrm{rms})\end{array}$ \\
\hline Eberl et al. [30] & SPECT-SPECT & Heart & Rigid & - & $S A D$ & $\mathbf{P}$ & $\begin{array}{l}3.1 \pm 1.7 \mathrm{~mm} \\
1.3^{\circ} \text { (rot) }\end{array}$ & mean \pm s.d. \\
\hline Slomka et al. [66] & SPECT-SPECT & Heart & Affine & - & $S A D$ & $\mathbf{P}$ & $\begin{array}{l}1.5 \mathrm{~mm}(\mathrm{x}, \mathrm{y}, \mathrm{z}) \\
2.0^{\circ} \text { (rot), } 5.3 \% \text { (size) }\end{array}$ & mean (max.) \\
\hline \multicolumn{9}{|l|}{ Mutual information } \\
\hline Carrillo et al. [38] & MR-MR & Abdom. & Rigid & - & MI & $\mathbf{L}$ & $(\mathrm{x}, \mathrm{y}, \mathrm{z}) 3.05 \mathrm{~mm}$ & mean \\
\hline $\begin{array}{l}\text { Object = Main object to } \\
\text { Trans. = Transformation } \\
\text { Struc. = Structures used } \\
\text { Method = Method used in } \\
\text { Valid. = Validation metho } \\
\text { Error: rot. = Rotational en } \\
\text { Error type: s.d. = Standar }\end{array}$ & $\begin{array}{l}\text { gistered. } \\
\text { od. } \\
\text { istration }(\mathrm{T}=\mathrm{Th} \\
\text { stration }(\mathrm{C}=\mathrm{cro} \\
=\text { Phantom, Pa. }\end{array}$ & $\begin{array}{l}\mathrm{L}=\mathrm{L} \\
\text { orrelatio } \\
\text { tient, } \mathrm{S}\end{array}$ & $\begin{array}{l}\mathrm{s}, \mathrm{HS}= \\
\mathrm{S}=\text { leas } \\
\text { Simulate }\end{array}$ & $\begin{array}{l}\text { surfaces). } \\
\text { res voxel } \\
\text { es, } M=1\end{array}$ & $\begin{array}{l}\text { nce, } M I=m \\
\text { gned images, }\end{array}$ & $\begin{array}{l}\text { nforr } \\
\text { andn }\end{array}$ & & \\
\hline
\end{tabular}

Landmarks have been exploited to estimate rigid registration error in [30], [31], [35], [37], and [38]. Savi et al. [39] rigidly registered cardiac PET and US images by using homologous anatomical landmarks (the two papillary muscles and the inferior junction of the right ventricle) of the heart. Rigid US-PET image registration was first performed in a plane identified by three landmarks. The obtained registration parameters were then applied to the whole PET volume. Sinha et al. [40] validated the rigid heart surface-based cardiac MR and PET image registration method by analyzing registration error using cardiac landmarks (papillary muscles, the insertion point of the right ventricle into the septum, the most inferior aspect of the septum, and the most inferior aspect of the lateral wall). Identification of landmarks was prone to errors because of their finite width and complex shapes. Especially in multimodal cardiac image registration, the accurate localization of the same anatomical landmarks can be difficult. Sometimes automatically detected points and lines can be used as landmarks [41]. Wirth et al. [42] utilized landmark-based elastic registration to register a thorax MR image to the coordinates of a CT image of the same subject. In this method, 36 corresponding landmarks in thorax and lung surfaces were elastically matched, and the rest of the points were mapped using interpolation or elastic mapping functions. The method registered corresponding points well, but general validation of the method accuracy was not performed.

2) Edge- and Surface-Based Registration: The chamfer matching method [43], [44] is often used to register surfaces and point sets. In this method, the sum of the distances between the transformed points and a distance map built upon the segmented surface using the chamfer distance transformation is minimized [45]. For cardiac image registration, chamfer 
matching methods have mainly been utilized for rigid registration methods based on the thorax structures [26], [31], [46], [47]. Also the ICP algorithm of Besl et al. [48] has been used to elastically register surfaces and lines [49], [50]. In the ICP method, the distances between structures are explicitly computed at every iteration of the registration algorithm and the sum of distances minimized. The "head-and-hat" algorithm [51], [52] has also been commonly proposed to register medical images and was first presented to register brain images. This algorithm models the contours from one of the images (usually higher resolution) as a surface (the "head") and the contours of the other image set as a series of points (the "hat"). The algorithm then determines the optimum rigid transformation, which minimizes the mean squared deviation between the points of the hat and the surfaces of the head by using the Powell minimization algorithm [53], [54]. The "head-and-hat" method has been applied for registering surfaces from cardiac MR and PET images [40] and thorax CT and PET images [33].

a) Registration methods based on thorax surfaces: Cardiac image registration methods based on the registration of the thorax surfaces have been proposed because it is often difficult to extract structural information from the heart surfaces directly. Thorax and lung surfaces are, in general, well visible in MR and CT images and in PET and SPECT transmission images. In the registration methods based on the thorax surfaces, every surface point that is involved in registration should have a unique corresponding point in the other image. In practice, this usually means that the axial extension in the reference study must be greater than in the study to be matched [18]. Sometimes, artificial edges of the images have to be excluded from the registration parameters' calculation. Surfaces from the transmission images have been utilized for intramodality registration of cardiac PET images [26] and for intermodality registration of cardiac PET and SPECT images [31], MR and PET images [47], and thorax CT and PET images [33], [46], [55]. Thorax and lung surfaces have often been obtained using the simple thresholding method of Yu et al. [33], where a threshold value of $50 \%$ of the maximum soft-tissue value was selected to segment the PET and SPECT transmission images [31], [46] and thorax CT images [33], [46]. Also, deformable models have been applied to segment thorax structures from PET transmission and MR images [47]. In this rigid registration method, chamfer matching was used to register segmented surfaces from PET transmission image with the MR transaxial image. Also, SA PET images were calculated from registered transaxial images by using header information between MR transaxial and SA images.

b) Registration methods based on heart surfaces: Registration of the heart surfaces may result in better registration of the area of interest [31]. The choice of the surfaces to be registered (e.g., epicardial and/or endocardial) is important. Faber et al. [29] presented a method for the intersubject rigid registration of 4-D gated cardiac SPECT perfusion images to the coordinates of the gated MR image. Left ventricular ED and ES endocardial surfaces were automatically detected from both image modalities using a model-based surface detector [56]. The best single transformation was searched to register SPECT ED and ES surfaces with corresponding surfaces in an MR image. Registration of the center of mass of the surfaces was used as an initial align- ment, and the "head-and-hat" algorithm [51], [52] was used to find more accurate registration parameters. After registration, quadrilinear interpolation was applied to the SPECT image to obtain a temporal correlation with time frames of an MR image. Sinha et al. [40] presented a method to register gated cardiac FDG PET images to the coordinates of gated MR images. In this method, contours of the left ventricular wall were defined from both imaging modalities by using an interactive algorithm with morphologic operators. Registration parameters were defined by using the "head-and-hat" surface matching approach [51]. In the MunichHeart software [57], endocardial and epicardial contours were manually delineated from SA MR images and registered with the same contours extracted from PET or SPECT images using the maximum count detection algorithm [58]. Declerck et al. [49], [50] presented an automated elastic registration method to align images from rest and stress myocardial perfusion SPECT studies. In this method, feature points of the cardiac SPECT image surfaces were extracted using a Canny-Deriche edge detector [59], [60]. The features were then registered using the ICP method [48]. Images were also elastically registered with a template image by using local spline transformations. The method was also applied to cardiac SPECT perfusion followup studies [61]. Thirion et al. [62], [63] presented a deformable model-based elastic registration method for intramodality registration of diastolic and systolic CT or SPECT images. Andersson et al. [64] utilized heart edge information for the cardiac PET emission images to rigidly reduce movement artifacts. The method was also applied to rigidly realign cardiac PET emission and transmission images [65].

\section{B. Registration Methods Based on Voxel Similarity Measures}

Registration methods based on voxel similarity measures can be divided into methods based on moments and principal axes, intensity difference and correlation methods, and methods based on mutual information.

1) Methods Based on Moments and Principal Axes: Image registration methods based on moments and principal axes use statistical factors derived from image data [7]. Moments describe the spatial distribution of the mass (intensity) of the image. Methods based on the principal axes register images by bringing the principal axes of the inertia tensors of corresponding objects in the images into coincidence. Accurate registration based on principal axes requires that the entire object be present in both imaging sets. Therefore, applications of these methods are limited. The principal-axes approach has been used to initially register myocardial SPECT stress and rest scans to templates [66], using SAD and SSC methods (see Section II-B2) to obtain more accurate registration. For the registration of thorax images, the principal-axes approach was proposed as an initial registration between $\mathrm{CT}$ and transmission SPECT images [32], while SAD and VIR measures (see Section II-B2) were applied to obtain the final registration.

2) Intensity Difference and Correlation Methods: Image intensity difference and correlation methods attempt to determine the best registration by maximizing the similarity between images that differ primarily because of different image-acquisition conditions, like noise [7]. The assumption of these methods is usually that pixel values in the registered images are strongly 
correlated. Therefore, these methods are particularly powerful for intramodality registration methods.

a) Intramodality registration: Hoh et al. [67] compared the SAD and SSD similarity measures for the rigid registration of cardiac PET emission images. In the SAD method, registered images are subtracted pixel-by-pixel, and the mean value of the sum of the absolute intensity difference of all the pixels in the subtracted image is computed. The SSD is similar to the SAD measure, but the squared intensity difference is calculated instead of the absolute difference. The SSD is the optimum measure when registered images differ only by Gaussian noise [12], [68]. In the paper by Hoh et al. [67], the effect of various defects and misalignments was simulated. No significant differences in the translation or rotation errors of the SAD and SSD algorithms were found. Slomka et al. [66] compared the SAD and SSC methods for affine registration of SPECT emission images to templates. An initial registration was obtained using alignment of the principal axes. Registered images were first subtracted, and the SSC was determined by counting along each pixel row the number of times the pixel gray level in the subtracted images went from negative to positive or from positive to negative [67]. At the optimum registration, there is a maximum of total sign changes. Slomka et al. [66] argued that the SAD provided better results than the SSC. This method was later enhanced and proposed for voxel-by-voxel quantification of SPECT images as a clinical tool [69]. In the enhanced method, not only did the registration algorithm compensate for shape differences by affine registration, but also a template erosion technique was used (role similar to warping adjustments) for fine tuning of the registration. The SSD-based similarity measure has also been applied in rigid motion correction (caused mainly by breathing) of gated heart perfusion MR images [37]. Perfusion MR imaging often takes more than $3 \mathrm{~min}$. Breath holding is not possible during the imaging protocol, nor can respiratory gating be used since a high temporal resolution is needed. Therefore, dynamic gated heart images and temporal resolution are degraded by respiratory-induced movements during the whole sequence [37]. In the recent paper by Klein et al. [23], [70], a novel affine 4-D registration algorithm was proposed for motion compensation of gated cardiac PET emission images to give better estimation of perfusion and metabolic parameters. The method registers different cardiac PET emission image time frames with the end- diastolic time frame. It uses nonuniform elastic material model (12-parameter global affine motion model) and iteratively calculates registration parameters of the model using a cost function that combines a least squares voxel difference measure with penalty terms assuming constant velocity and an affine model. The method does not require the precise a priori segmentation of the object.

Turkingston et al. [71] utilized cross-correlation measure for the rigid alignment of dynamic cardiac PET images to cardiac templates. The method used only translations, assuming that the orientation of the heart remains the same during the study. The cross-correlation technique has also been proposed for rigid motion correction of cardiac SPECT images [72], [73]. Bettinardi et al. [74] utilized the cross-correlation measure to rigidly register two PET transmission images for patient repositioning. Cross-correlation measure was also used for the correction of the patient motion in the PET heart studies with the help of PET transmission images, taken before and after emission imaging [74]. Gallippi et al. [75] utilized modified correlation measure to match local brightness statistics of the registered images. The method was applied to rigidly register intramodality cardiac MR or US time series images. Bacharach et al. [76] utilized CC measure to rigidly register two cardiac PET emission scans of the same subject acquired at different times. The method was based on the registration of corresponding transmission data sets. The optimum alignment was defined as the one that produced the maximum value of the $\mathrm{CC}$ between the two data sets. $\mathrm{CC}$ is an optimal measure for registration in the case of a linear relationship between the intensity values in the images to be registered [11], [12], [68], [77]. This is seldom the case between different image modalities, and the $\mathrm{CC}$ is thus mainly used for intramodality registration.

b) Intermodality registration: In the paper by Dey et al. [32], SAD and VIR methods were compared for the rigid registration of thorax CT and SPECT images. In the VIR method, the sum of the normalized standard deviations is calculated to define registration parameters. The method was first proposed by Woods et al. [78], [79] for registering intramodality brain PET images [78] and intermodality brain PET and MR images [79]. In the latter, the VIR algorithm minimizes the normalized standard deviation of PET voxel values for each MR intensity value, but the method could also be used for these images vice versa [12]. Dey et al. [32] utilized SPECT transmission image as a linking mediator to register thorax CT and SPECT emission images. In this VIR method, the Simplex algorithm was applied for the minimization of the cost function, while in the original VIR method [79], the Newton-Rhapson method [54] was used. An approximate image alignment was made using a technique based on the principal-axes transformation [80]. VIR provided better convergence than SAD and may perform better for CT and SPECT image registration, but the method was only tested on phantom images. In the paper by Eberl et al. [30], the SAD, SSC, VIR, and sum of pixel-by-pixel product measures were compared for rigid registration of intramodality cardiac SPECT emission images or intermodality cardiac PET and SPECT emission images. SAD was recognized to be the most accurate and reliable method. The use of SAD and VIR thus depends on the type of images to be registered.

In [81], the cross-correlation measure was utilized to rigidly register cardiac MR and PET emission images by using PET transmission image as a linking mediator for registration. Edge information and a region growing algorithm were combined to segment lung cavities from both MR and PET transmission images. The segmented cavities were then utilized as landmarks for registration. Matching of the center of mass of segmented cavities was exploited as an initial registration, and the crosscorrelation function was employed for maximizing overlapping areas of the lung cavities for accurate registration. The method was validated only qualitatively, using visual inspection.

3) Mutual Information: Mutual information is an information theory measure of the statistical dependence between two random variables or the amount of information that one variable contains about the other [11], [12], [82]-[84]. Mutual information can be qualitatively considered as a measure of how well one image explains the other. The mutual information is maxi- 
mized at the optimal alignment [77]. No assumptions are made regarding the nature of the relation between the image intensities in the registered images [82]. Therefore, the mutual information method is promising in particular for intermodality registration.

Intermodality registration differs from intramodality registration because different medical imaging modalities usually have different intensity characteristics and different resolutions, noise characteristics, and fields of view. Several normalized versions of the mutual information has been proposed because changes in overlap of very low-intensity regions of the image can disproportionately contribute to the mutual information measure [10]-[12].

Registration based on mutual information has been proposed to register thorax CT and PET images, rigidly [85] and elastically [86]. In the latter, the rigid registration method [85] was first applied as an initialization prior to the elastic registration. A nonlinear thin-plate-spline warping was done using lung contours detected on PET transmission scans and CT volumes. Nonlinear deformation significantly improved the alignment of PET with breath-hold CT. Carrillo et al. [38] used mutual information,VIR, and the CC methods for registering abdominal thorax MR images. Results were compared with the manual registration method. The best registration results were obtained using the mutual information with the Powell minimization algorithm. In [87], a method that associates mutual information, gradient information, and the smoothness of the registration transformation was presented to elastically register intrapatient cardiac $\mathrm{MR}$ and PET images. A rigid thorax surface-based cardiac registration method [47] was used for the initial registration of the images.

\section{Implementation: Interpolation and Optimization}

In a registration process, the image interpolation and minimization algorithm are key points. We give hereafter some comments on these important topics.

1) Interpolation: Interpolation is required when an image needs to be translated, rotated, scaled, warped, or otherwise deformed before it can match a reference image or an atlas [88]. In volumetric imaging, interpolation is often used to compensate for nonisotropic data sampling. This is typically the case with cardiac and thorax images where the in-slice resolution can be much higher (e.g., $1 \mathrm{~mm}$ ) than the interslice resolution (e.g., $8 \mathrm{~mm}$ ). In intermodality registration, one image may be of substantially lower resolution than the other, and in cardiac image registration, lower resolution images (e.g., SPECT or PET) are often transformed to the sample space of the higher resolution modality (e.g., CT or MR) [29], [40]. To obtain the same isotropic voxel dimension in cardiac and thorax image registration, trilinear interpolation is often used [47], [88]. Nekolla et al. [57] created a scaled isotropic set from individual SA MR and PET images using a cubic interpolation. In [37], bicubic interpolation was used for interpolation of SA MR perfusion images. Faber et al. [29] used quadrilinear interpolation to interpolate a 4-D SPECT image to the coordinates of a corresponding MR image. For cardiac nuclear medicine images, cubic convolution interpolation method has been recognized as efficient [89].

2) Optimization: For rigid 3-D image registration, the optimal transformation usually minimizes a cost function with six degrees of freedom (three translations and three rotations), giving a six-dimensional parameter space. Elastic registration algorithms have more degrees of freedom, in which case the parameter space has correspondingly more dimensions [10]-[12]. Because the heart is a nonrigid moving object, elastic registration is ideally needed. An exhaustive search to find the global minimum of the cost function is usually computationally too extensive and time consuming. Nonoptimal optimization methods, like Powell [53], [54] and Simplex [54], [90] methods, are applied to find an optimum faster than with the exhaustive search. The Powell method has been selected for the minimization of cardiac image registration methods in [29], [33], [38], and [46] and for thorax image registration methods in [32], [85], and [86]. The Simplex method has been used for cardiac image registration in [30], [66], [67], and [69].

Multiresolution methods can be implemented to increase the probability of finding the global optimum in the parameter space and to make the registration procedure faster. In the multiresolution approach, the images are first registered at a low resolution, and the transformation solution is applied to the next resolution level. The process is repeated until the highest resolution level is reached. For cardiac image registration, the multiresolution approach has been applied in [26], [37], [47], and [86]. To our knowledge, no comparative studies on the performance of minimization methods for cardiac image registration have been published to date.

\section{VALIDATION OF REGISTRATION MEthodS}

A method can not be accepted as a clinical tool without careful validation. Validation of registration accuracy is a difficult task because the ground truth (i.e., gold standard) is generally not available [11], [12], [91]. Registration methods are often validated using external markers, anatomical landmarks, or external fiducial frames as the gold standards [91]. Visual inspection is the most obvious method for evaluation of the registration accuracy but can be considered as an informal and insufficient approach.

A direct comparison of the measurements reported in the literature is not straightforward because of the nonunique definition of accuracy and of the different methods adopted to measure it [31]. In cardiac image registration, the main interest is the registration accuracy in the heart area (target registration error) [12]. The mean and rms errors are commonly used measures for registration errors [11], [12], [91]. In rigid-body registration, error in the parameters of the spatial transformation model, such as errors in $x$-axis translation, are also commonly reported. However, the decomposition of a rigid-body movement into elementary rotations and translations is not unique, i.e., the result depends on the order of the elementary operations [92].

To reduce registration errors caused by cardiac movement and respiration, ECG gating and breath holding (or breath gating) are sometimes used. The problem for cardiac image registration of ECG-gated cine MR images is often that the same anatomical region is not observed within the same slice of the cine images. Recent MRI "slice following" techniques should make temporal registration easier, showing the same anatomical locations of the heart through cine image sequence. In thorax surface-based 
cardiac registration methods, movement of the thoracic wall and diaphragm is quite different in magnitude in all directions. Movements can lead to distortions and asymmetrical position changes that can cause errors while determining the registration parameters [93]. The movements near the diaphragm are largest, reaching several centimeters [94]. Cardiac PET and SPECT images are often integral images through time (static images). This causes extra difficulties for registering, e.g., with gated MR images. In the case where both registered images are integral images through time, the errors caused by breathing and cardiac motion can be considered to be similar in both images [31].

In registration methods in which a transmission image (PET, SPECT) is used as a linking mediator to register corresponding emission image, the assumption is that the patient does not move during and between image acquisition. Because the image-acquisition times in cardiac PET and SPECT transmission and emission images are often several minutes, movement artifacts often occur. In modern PET imaging scanners, emission and transmission images are acquired without taking the patient out of the scanner between acquisitions. Yu et al. [95] argue that careful application of laser alignment is an adequate method of registration in the PET imaging systems where the patient is taken out of the scanner between transmission and emission acquisitions during the uptake period. Methods have also been presented for the rigid registration of PET emission and transmission images [74], [96]. If the movement between SPECT transmission and the emission image is more than $2-3 \mathrm{~cm}$, it can also seriously affect the attenuation correction of the emission image and, thus, its quality [97].

\section{A. Phantom Studies}

Phantom studies are important for the estimation of the registration accuracy because a phantom can remain perfectly still and can be displaced and sometimes even rotated with considerable accuracy. Phantom-based validation is utilized especially for estimating the accuracy of intramodality registration methods. For registration methods based on thorax surfaces, the registration accuracy has been usually validated using thorax phantoms [26], [30]-[33], [35], [46], [74]. For example, the Alderson thorax phantom [31], [98], a physical torso phantom with lungs, cardiac, and spine inserts (Data Spectrum) [32] and Jaszczak thorax phantom (Data Spectrum) [35] have been applied. In [71], a heart phantom was used. Simulations of images and different error sources can be used to estimate cardiac registration accuracy [26], [55]. Klein et al. [23], [70] utilized a mathematical cardiac phantom [99] to validate a 4-D motion correction algorithm of cardiac PET images. Integrated imaging devices such as combined PET/CT scanners [100], [101] could also provide gold standards for registration [94].

The accuracy of thorax surface-based registration methods depends on the modalities and structures to be registered. Pallotta et al. [26] compared the registration accuracy of the method where segmented PET transmission images were linking mediators to register corresponding PET emission images. A synthetic thorax phantom was used to validate the registration accuracy by using only external thorax surfaces (E), internal lung surfaces (I), or both thorax and lungs (EI). Seven markers were positioned to the external surfaces of the synthetic thorax phantom.
Average values for the mean residual marker displacement over ten experiments were $3.41 \pm 1.41 \mathrm{~mm}, 2.27 \pm 0.76 \mathrm{~mm}$, and $2.19 \pm 0.52 \mathrm{~mm}$ for E, I, and EI surfaces, respectively. The rotational error was smaller than $1.5^{\circ}$ in each case. The result indicated that the more the surfaces were integrated in the registration, the more accurate the result.

\section{B. Registration Accuracy}

\section{1) Intramodality Registration:}

a) MRI: Bidaut et al. [37] rigidly registered intramodality gated heart perfusion MR images by using SSD measure and obtained $3.0-\mathrm{mm}$ accuracy in $x, 1.6-\mathrm{mm}$ accuracy in $y$, and 2.2-mm accuracy in $z$ directions. Gallippi et al. [75] utilized correlation measure and warping to register cardiac MR time-series images. Mean left-right registration error of $1.23 \pm 0.06 \mathrm{~mm}$ and mean anterior-posterior error of $3.25 \pm 1.04 \mathrm{~mm}$ were reported. With intramodality mutual information-based registration of abdominal MR images, Carrillo et al. [38] reported 3-mm accuracy by using anatomical landmarks.

b) PET: Hoh et al. [67] registered cardiac PET emission images using SAD and SSC measures. For both methods, accuracy was typically $0.5 \pm 0.5 \mathrm{~mm}$ in the inplane direction, $1.1 \pm 1.1 \mathrm{~mm}$ in the interplane direction, and $0.9 \pm 1.1^{\circ}$ for all rotational directions. Turkingston et al. [71] used cross-correlation measure for the alignment of dynamic cardiac PET emission scans to templates and showed that the rigid registration technique was reliable within one voxel $(1.7 \times 1.7$ $\times 4.2 \mathrm{~mm}^{3}$ ). Pallotta et al. [26] obtained with synthetic thorax phantom $2.19 \pm 0.52 \mathrm{~mm}$ rms error for rigid registration of cardiac PET emission images with $1.5^{\circ}$ rotational error, while using both thorax and lung surfaces from corresponding transmission images for registration. Klein et al. [23], [70] reported a 1.9-, 2.4-, and 6.8-mm maximum registration error for the $x, y$, and $z$ directions, respectively, after the use of a 4-D PET motion compensation algorithm. For theCC-based PET transmission image registration of corresponding cardiac PET emission images, Bacharach et al. [76] reported 1-mm accuracy in $x, y$, and $z$ directions and $1.5^{\circ}$ in the three angles of rotation.

c) SPECT: Eberl et al. [30] rigidly registered intramodality SPECT emission images by using SAD measure and obtained $2.1 \pm 1.2 \mathrm{~mm}$ accuracy by using a phantom experiment.

\section{2) Intermodality Registration:}

a) MR-PET: Sinha et al. [40] reported a $1.95 \pm 1.6 \mathrm{~mm}^{2}$ accuracy for a rigid heart surface-based registration method of ECG-gated cardiac MR and FDG PET images. This error was estimated only in the $(x, y)$ plane using 80 internal landmarks from six volunteer scans. In [47], a $2.8 \pm 0.5 \mathrm{~mm}$ error was reported for the rigid registration of cardiac MR and PET images. The reported error was a surface distance registration error between thorax and lung surfaces. The measure depended also on deformable model-based segmentation results, but provided also a reasonable measure of the registration accuracy of the MR and PET cardiac (thorax) images.

b) MR-SPECT: For rigid heart surface-based registration of MR and SPECT images, Faber et al. [29] reported a 2.7- $\mathrm{mm}$ registration accuracy. 
c) CT-PET: In the rigid thorax and lung CT and PET image registrations, where a PET transmission image was used as a linking mediator to register PET emission image to CT image coordinates, accuracy of $1-3 \mathrm{~mm}$ in the transaxial plane and $2-4 \mathrm{~mm}$ in the longitudinal direction was reported in [33], [46], and [55], with about $1.5^{\circ}$ rotational error [46].

d) CT-SPECT: For the rigid registration of thorax $\mathrm{CT}$ and SPECT emission images with the help of SPECT transmission images, the VIR and SAD methods were found to have about the same accuracy (about $3.5 \mathrm{~mm}$ ), but VIR provided better convergence [32].

e) PET-SPECT: For rigid cardiac PET and SPECT image registration based on the segmentation of thorax and lung surfaces from transmission images, the accuracy was reported to be on the order of $3 \mathrm{~mm}$ in longitudinal direction and $5 \mathrm{~mm}$ in the transaxial plane [31]. For rigid cardiac PET and SPECT emission image registration based on SAD measure, Eberl et al. [30] reported $3.1 \pm 1.7 \mathrm{~mm}$ accuracy. Nekolla et al. [57] rigidly registered heart surfaces from PET and SPECT images, and a mean distance between the two registered heart surfaces was less than $2.5 \mathrm{~mm}$. The surface distance error measure depended on segmentation results but provided a reasonable measure of the registration accuracy.

\section{CONCLUSION}

Registration of different cardiac imaging modalities is the preliminary and mandatory step to combining anatomical and functional cardiac information. The integration of multiple complementary data into a common reference allows a more comprehensive analysis of the cardiac functions and pathologies. The accurate spatial coregistration of different imaging modalities also provides additional useful clinically relevant information, or information relevant to cardiac research, which is not available while looking at images from a single modality.

We have presented a survey of various cardiac image registration methods, which were coarsely divided into registration methods based on geometric image features and voxel similarity measures. In the first category, registration relies on the extraction of geometric features; in the latter, preliminary extraction of the features is not needed.

The choice of a cardiac registration method is difficult since, at the present time, no general fully automatic method exists that could handle the wide variety of encountered clinical situations (modalities, acquisition protocols, etc.). Moreover, it should also be driven by the evaluation of the methods' performances with the same common databases for which the ground truth is available. Such a reference does not exist either. We give hereafter some critical comments about the main categories of cardiac image registration methods.

1) External skin marker-based registration of cardiac images does not guarantee registration of the heart within the body, since heart position changes with body position (e.g., prone or supine), respiration, and cardiac contraction [29]. Also, skin markers cannot be utilized retrospectively.

2) Landmark-based registration of the heart is also difficult because there are few spatially accurate anatomical land- marks in cardiac images. Landmarks can also be less visible with certain modalities and in some pathological conditions, such as ischemia.

3) Thorax surface-based methods can be used if it is not possible to obtain structural information from the heart surfaces directly. In thorax surface, based cardiac image registration methods, it is recommended to use both thorax and lung surfaces, which are well visible in thorax MR and CT images and in PET and SPECT transmission images [26]. Still, these methods are prone to errors induced by respiration and different movement artifacts [94].

4) Registration of the heart surfaces directly will result in the better registration of the area of interest. The choice of the surfaces to be registered (e.g., epicardial and/or endocardial) is important and depends on the application and modalities to be used. Gated acquisitions combined with breath-hold (or breath-gated) image acquisition [94] give in many cases acceptable results even with rigid cardiac registration methods.

5) The voxel similarity measures, compared to geometric image feature-based registration methods, have the important advantage that they do not require a priori extraction of the registered features (e.g., segmentation). The use of image intensity difference and correlation methods relies on the assumption that pixel values in the registered images are strongly correlated. This is usually not the case with intermodality registration. In modern information-theoretic voxel similarity methods, like mutual information, no assumptions are made regarding the nature of the relation between the image intensities in the registered images [82]. These methods are particularly promising for the intermodality cardiac image registration. Because of the recent development of the mutual information-based methods, applications to cardiac image registration are still rare. One of the aims of recent research in the voxel similarity measure-based registration area has been to devise general algorithms that will work on a wide variety of image types, without application-specific preprocessing [12].

Rigid cardiac image registration generally does not describe the spatial relationship between images adequately. Elastic (nonrigid) cardiac image registration is needed especially because of cardiac motion; between end-diastole and end-systole (during cardiac cycle), the heart valvular plane moves 9$14 \mathrm{~mm}$ toward the apex and the myocardial walls thicken from approximately 10 to over $15 \mathrm{~mm}$ [23], [102], [103]. Also, the problems due to imaging conditions, different movement artifacts, and elasticity of the body, lungs, and heart cause different tissue deformations that are not possible to compensate for using rigid registration methods. There is considerable research going on in extending the use of intensity-based registration algorithms to nonrigid transformations [12].

Deformable model-based approaches (deformable registration algorithms) for cardiac image registration are particularly promising for elastic 4-D registration of the cardiac images (e.g., to compensate for movement artifacts) [23], [70]. Model-based approaches can also lead to the integration of information from different imaging modalities into an individualized heart model, 
including anatomical and functional information [69], [104]. This kind of model can be applied in computer-assisted diagnosis, treatment planning, and surgery. Data fusion techniques, based, i.e., on neural networks and fuzzy logic, can be used to interpret and summarize the large amount of information from registered images and to help establish a diagnostic or select a therapy [4], [105]. Methods combining similarity measures with a priori knowledge from geometric models can also provide new possibilities, especially for elastic registration [87], [106].

The validation of the registration accuracy is particularly important. Virtual and physical phantoms may provide the gold standard for validation. Also, image databases may in the future provide a source for the objective comparison of different cardiac registration methods.

Cardiac image registration remains a challenge because of the numerous specific problems mainly related to the different motion sources (patient, respiration, heart) and to the specificity of each imaging modality. Up to now, no general method is able to automatically register any modality with any other modality. Cardiac image registration methods also always require a compromise among accuracy, precision, reliability, robustness, and issues such as automation, interactivity, speed, patient-friendliness, etc. It is also important to keep in mind that the registration techniques and results should be useful and usable in clinical practice.

\section{ACKNOWLEDGMENT}

The authors are grateful to the anonymous reviewers for their suggestions to improve this paper.

\section{REFERENCES}

[1] J. Hartiala and J. Knuuti, "Imaging of heart by MRI and PET," Ann. Med., vol. 27, pp. 35-45, 1995.

[2] N. Wilke, M. Jerosch-Herold, A. E. Stillman, K. Kroll, N. Tsekos, H. Merkle, T. Parrish, X. Hu, Y. Wang, J. Bassingthwaighte, R. J. Bache, and K. Ugurbil, "Concepts of myocardial perfusion imaging in magnetic resonance imaging," Magn. Res. Quart., vol. 10, pp. 249-286, 1994.

[3] H. R. Schelbert, "Metabolic imaging to assess myocardial viability," $J$. Nucl. Med., vol. 35, pp. 8-14, 1994.

[4] F. Behloul, B. P. F. Lelieveldt, A. Boudraa, M. Janier, D. Revel, and J. H. C. Reiber, "Neuro-fuzzy systems for computer-aided myocardial viability assessment," IEEE Trans. Med. Imag., vol. 20, pp. 1302-1313, Dec. 2001.

[5] F. Behloul, M. Janier, P. Croisille, C. Poirier, A. Boudraa, R. Unterreiner, J. Mason, and D. Revel, "Automatic assessment of myocardial viability based on PET-MRI data fusion," in Int. Conf. IEEE Engineering in Medicine and Biology Soc., 1998, pp. 429-495.

[6] G. D. Waiter, A. Al-Mohammad, M. Y. Norton, T. W. Redpath, A. Welch, and S. Walton, "Regional myocardial wall thickening assessed at rest by ECG gated (18)F- FDG positron emission tomography and by magnetic resonance imaging," Heart, vol. 84, pp. 332-333, 2000.

[7] C. Maurer and J. M. Fizpatrick, "A review of medical image registration," Interact. Image-Guided Neurosurg., pp. 17-44, 1993.

[8] J. B. A. Maintz and M. A. Viergever, "A survey of medical image registration," Med. Image Anal., vol. 2, no. 1, pp. 1-36, 1998.

[9] P. A. van den Elsen, E.-J. D. Pol, and M. A. Viergever, "Medical image matching-A review with classification," IEEE Eng. Med. Biol., vol. 12, no. 2, pp. 16-39, 1993.

[10] D. L. G. Hill, P. G. Batchelor, M. H. Holden, and D. J. Hawkes, "Medical image registration," Phys. Med. Biol., vol. 46, no. 1, pp. 1-45, 2001.

[11] J. V. Hajnal, D. L. G. Hill, and D. J. Hawkes, Medical Image Registration. Boca Raton, FL: CRC Press, 2001.

[12] J. M. Fitzpatrick, D. L. G. Hill, and C. R. Maurer, Handbook of Medical Imaging. Bellingham, WA: SPIE Press, 2000, vol. 2, ch. Image Registration, pp. 375-435.
[13] I. N. Bankman, Handbook of Medical Imaging: Processing and Analysis: Academic Press, 2000.

[14] A. F. Frangi, W. J. Niessen, and M. A. Viergever, "Three-dimensional modeling for functional analysis of cardiac images, a review," IEEE Trans. Med. Imag., vol. 20, pp. 2-25, Jan. 2001.

[15] M. Audette, F. Ferrie, and T. Peters, "An algorithmic overview of surface registration techniques for medical imaging," Med. Image Anal., vol. 4, no. 4, pp. 201-217, Feb. 2000.

[16] H. Lester and S. R. Arridge, "A survey of hierarchical nonlinear medical image registration," Pattern Recognit., vol. 32, pp. 129-149, 1998.

[17] L. G. Brown, "A survey of image registration techniques," $A C M$ Comput. Surv., vol. 24, no. 4, pp. 325-376, 1992.

[18] M. C. Gilardi, G. Rizzo, A. Savi, and F. Fazio, "Registration of multimodal biomedical images of the heart," Q. J. Nucl. Med., vol. 40, no. 1, pp. 142-150, 1996.

[19] A. W. Habboosh, "A review of MRI and PET correlation," in Proc. IEEE Conf. Bioengineering, 1992, pp. 16-17.

[20] G. Z. Yang, P. Burger, J. Panting, P. D. Gatehouse, D. Rueckert, D. J. Pennel, and D. N. Firmin, "Motion and deformation tracking for short-axis echo-planar myocardial perfusion imaging," Med. Image Anal., vol. 2, no. 3, pp. 285-302, Mar. 1998.

[21] J. C. McEachen II, A. Nehorai, and J. S. Duncan, "Multiframe temporal estimation of cardiac nonrigid motion," IEEE. Trans. Med. Imag., vol. 9, pp. 651-665, Apr. 2000.

[22] X. Papademetris, A. J. Sinusas, D. P. Dione, and J. S. Duncan, "Estimation of 3D left ventricular deformation from echocardiography," Med. Image Anal., vol. 5, pp. 17-28, 2001.

[23] G. J. Klein and R. H. Huesman, "Four-dimensional processing of deformable cardiac PET data," Med. Image Anal., vol. 6, pp. 29-46, 2002.

[24] T. R. Nelson, D. B. Downay, D. H. Pretorius, and A. Fenster, Three-Dimensional Ultrasound. Philadelphia, PA: Lippincott Williams \& Wilkins, 1999.

[25] K. S. Arun, T. S. Huang, and S. D. Blostein, "Least-squares fitting of two 3-D point sets," IEEE Trans. Pattern Anal. Machine Intell., vol. PAMI-9, pp. 698-700, May 1987.

[26] S. Pallotta, M. C. Gilardi, V. Bettinardi, G. Rizzo, C. Landoni, G. Striano, R. Masi, and F. Fazio, "Application of a surface matching image registration technique to the correlation of cardiac studies in positron emission tomography by transmission images," Phys. Med. Biol., vol. 40, pp. $1695-1708,1995$.

[27] Q. Li, L. Zamorano, Z. Jiang, F. Vinas, and F. Diaz, "The application accuracy of the frameless implantable marker system and analysis of related affecting factors," in Lecture Notes in Computer Science 1496: Medical Image Computing and Computer-Assisted Intervention, MICCAI98, W. M. Wells, A. Colchester, and S. Delp, Eds., 1998, pp. 253-260.

[28] O. Sipilä, "Methodological aspects for improving clinical value of SPECT and MRI," Ph.D. dissertation, Helsinki Univ. Technol., Helsinki, Finland, 2000.

[29] T. L. Faber, R. W. McColl, R. M. Opperman, J. R. Corbett, and R. M. Peshock, "Spatial and temporal registration of cardiac SPECT and MR images: Methods and evaluation," Radiology, vol. 179, no. 3, pp. 857-861, 1991.

[30] S. Eberl, I. Kanno, R. R. Fulton, A. Ryan, B. F. Hutton, and M. J. Fulham, "Automated interstudy image registration technique for SPECT and PET," J. Nucl. Med., vol. 37, no. 1, pp. 137-145, 1996.

[31] M. C. Gilardi, G. Rizzo, A. Savi, C. Landoni, V. Bettinardi, C. Rossetti, G. Striano, and F. N. Fazio, "Correlation of SPECT and PET cardiac images by a surface matching registration technique," Comput. Med. Imag. Graph., vol. 22, pp. 391-398, Dec. 1998.

[32] D. Dey, P. J. Slomka, L. J. Hahn, and R. Kloiber, "Automatic threedimensional multimodality registration using radionuclide transmission CT attenuation maps: A phantom study," J. Nucl. Med., vol. 40, no. 3 , pp. 448-455, 1999.

[33] J. N. Yu, F. H. Fahey, H. D. Gage, C. G. Eades, B. A. Harkness, and C. A. Pelizzari, "Intermodality, retrospective image registration in the thorax," J. Nucl. Med., vol. 36, no. 12, pp. 2333-2338, Dec. 1995.

[34] J. West, J. M. Fitzpatrick, M. Y. Wang, B. M. Dawant, C. R. Maurer Jr., R. M. Kessler, R. J. Maciunas, C. Barillot, D. Lemoine, A. Collingnon, F. Maes, P. Suetens, D. Vandermeulen, P. A. van den Elsen, S. Napel, T. S. Sumanaweera, B. Harkness, P. F. Hemler, D. L. G. Hill, D. J. Hawkes, C. Studholme, J. B. A. Maintz, M. A. Viergever, G. Malandain, X. Pennec, M. E. Noz, G. Q. Maguire Jr., M. Pollack, C. A. Pelizzari, R. A. Robb, D. Hanson, and R. P. Woods, "Comparison and evaluation of retrospective intermodality image registration techniques," J. Comput. Assist. Tomogr., vol. 21, pp. 554-566, 1997. 
[35] E. L. Kramer, M. E. Noz, J. J. Sanger, A. J. Megibow, and G. Q. Maguire, "CT-SPECT fusion to correlate radiolabeled monoclonal antibody uptake with abdominal CT findings," Radiology, vol. 172, no. 10, pp. 861-865, 1989.

[36] H. Loats, "CT and SPECT image registration and fusion for spatial localization of metastatic processes using radiolabeled monoclonals," $J$. Nucl. Med., vol. 34, pp. 562-566, 1993.

[37] L. M. Bidaut and J.-P. Vallee, "Automated registration of dynamic MR images for the quantification of myocardial perfusion," J. Magn. Res. Imag., vol. 13, pp. 648-655, 2001.

[38] A. Carrillo, J. L. Duerk, J. S. Lewin, and D. L. Wilson, "Semiautomatic 3-D image registration as applied to interventional MRI liver cancer treatment," IEEE Trans. Med. Imag., vol. 19, pp. 175-185, Mar. 2001.

[39] A. Savi, M. C. Gilardi, G. Rizzo, M. Pepi, C. Landoni, C. Rossetti, G. Lucignani, A. Bartonelli, and F. Fazio, "Spatial registration of echocardiographic and positron emission tomographic heart studies," Eur. J. Nucl. Med., vol. 22, no. 3, pp. 243-247, 1995.

[40] S. Sinha, U. Sinha, J. Czernin, G. Porenta, and H. R. Schelbert, "Noninvasive assessment of myocardial perfusion and metabolism: Feasibility of registering gated MR and PET images," Amer. J. Roentgenol., vol. 36, pp. 301-307, 1995.

[41] X. Pennec, N. Ayache, and J.-P Thirion, Handbook of Medical Imaging: Processing and Analysis. New York: Academic, 2000, ch. Landmarkbased registration using features identified through differential geometry, pp. 499-513.

[42] M. A. Wirth, C. Choi, and A. Jennings, "Point-to-point registration of nonrigid medical images using local elastic transformation methods," in IEEE Int. Conf. Image Processing and Its Applications, 1997, pp. $780-784$.

[43] G. Borgefors, "Distance transformation in digital images," Comput. Vision Graph. Image Process., vol. 48, pp. 344-371, 1986.

[44] — - "Hierarchical chamfer matching: A parametric edge matching algorithm," IEEE Trans. Pattern Anal. Machine Intell., vol. 10, pp. 849-865, Nov. 1988

[45] M. Van Herk, Handbook of Medical Imaging: Processing and Analysis. New York: Academic, 2000, ch. Image registration using chamfer matching, pp. 515-527.

[46] J. Cai, J. C. Chu, D. Recine, M. Sharma, C. Nguyen, R. Rodebaugh, A. Saxena, and A. Ali, "CT and PET lung image registration and fusion in radiotherapy treatment planning using the chamfer-matching method," Int. J. Radiat. Oncol. Biol. Phys., vol. 43, no. 4, pp. 883-891, 1999.

[47] T. J. Mäkelä, P. Clarysse, J. Lötjönen, O. Sipilä, K. Lauerma, H. Hänninen, E.-P Pyökkimies, J. Nenonen, J. Knuuti, T. Katila, and I. E. Magnin, "A new method for the registration of cardiac PET and MR images using deformable model based segmentation of the main thorax structures," in Lecture Notes in Computer Science 2208: Medical Image Computing and Computer-Assisted Intervention, MICCAI01, W. J. Niessen and M. Viergever, Eds., 2001, pp. 557-564.

[48] P. J. Besl and N. D. McKay, "A method for registration of 3-D shapes," IEEE Trans. Pattern Anal. Machine Intell., vol. 14, pp. 239-256, Feb. 1992.

[49] J. Declerck, J. Feldmar, M. L. Goris, and F. Betting, "Automatic registration and alignment on a template of cardiac stress and rest SPECT images," INRIA, Res. Rep. 2770, 1996.

[50] - "Automatic registration and alignment on a template of cardiac stress and rest reoriented SPECT images," IEEE Trans. Med. Imag., vol. 16, pp. 727-737, Dec. 1997.

[51] C. A. Pelizzari, G. T. Y. Ghen, D. R. Spelbring, R. R. Weichselbaum, and C. T. Chen, "Accurate three-dimensional registration of CT, PET and/or MR images of the brain," J Comput. Assist. Tomogr., vol. 13, no. 1, pp. 20-26, 1989.

[52] D. N. Levin, C. A. Pelizzari, G. T. Y. Chen, C.-T. Chen, and M. D. Cooper, "Retrospective geometric correlation of MR, CT and PET images," Radiology, vol. 169, no. 3, pp. 817-823, 1988.

[53] M. J. Powell, "An iterative method of finding stationary values of a function of several variables," Comp. J., vol. 5, pp. 147-151, 1962.

[54] W. H. Press, S. A. Teukolsky, W. T. Vetterling, and B. P. Flannery, Numerical Recipes in C: The Art of Scientific Computing, 2nd ed. Cambridge, U.K.: Cambridge Univ. Press, 1992.

[55] Y.-C. Tai, K. P. Lin, C. K. Hoh, S. C. Huang, and E. J. Hoffman, "Utilization of 3-D elastic transformation in the registration of chest X-ray CT and whole body PET," IEEE Trans. Nucl. Sci., vol. 44, pp. 1606-1612, Aug. 1997.

[56] T. L. Faber, E. M. Stokely, R. M. Peshock, and J. R. Corbett, "A model based four-dimensional left ventricular surface detector," IEEE Trans. Med. Imag., vol. 10, pp. 321-329, Sept. 1991.
[57] S. Nekolla, T. Ibrahim, T. Balbach, and C. Klein, Understanding Cardiac Imaging Techniques-From Basic Pathology to Image Fusion. Amsterdam, The Netherlands: IOS Press, 2001, vol. 322, ch. Coregistration and fusion of cardiac magnetic resonance and positron emission tomography studies, pp. 144-154.

[58] S. Nekolla, C. Miethaner, N. Nguyen, S. Ziegler, and M. Schwaiger, "Reproducibility of polar map generation and assessment of defect severity and extent assessment in myocardial perfusion imaging using positron emission tomography," Eur. J. Nucl. Med., no. 25, pp. 1313-1321, 1998.

[59] J. Canny, "A computational approach to edge detection," IEEE Trans. Pattern Anal. Machine Intell., vol. PAMI-8, pp. 679-698, Nov. 1986.

[60] R. Deriche, "Using Canny's criteria to derive a recursively implemented optimal edge detector," Int. J. Comput. Vision, vol. 1, pp. 167-187, 1987.

[61] J.-P Thirion, Understanding Cardiac Imaging Techniques - From Basic Pathology to Image Fusion. Amsterdam, The Netherlands: IOS Press, 2001, ch. Perfusion and motion from gated SPECT, pp. 84-93.

[62] — , "Fast non-rigid matching of 3-D medical images," INRIA, Res. Rep. 2547,1995

[63] — , "Image matching as a diffusion process: An analogy with Maxwell's demons," Med. Image Anal., vol. 2, no. 3, pp. 243-260, 1998.

[64] J. L. R. Andersson, "A rapid and accurate method to realign PET scans utilizing image edge information," J. Nucl. Med., vol. 36, no. 4, pp. $657-669,1995$.

[65] J. L. R. Andersson, B. E. Bagnhammar, and H. Schneider, "Accurate attenuation correction despite movement during PET imaging," J. Nucl. Med., vol. 36, no. 4, pp. 670-678, 1995.

[66] P. J. Slomka, A. H. Gilbert, J. Stephenson, and T. Cradduc, "Automated alignment and sizing of myocardial stress and rest scans to three-dimensional normal templates using an image registration algorithm," J. Nucl. Med., vol. 36, pp. 1115-1122, 1995.

[67] C. K. Hoh, M. Dahlbom, G. Harris, Y. Choi, R. A. Hawkins, M. E. Philps, and J. Maddahi, "Automated iterative three-dimensional registration of positron emission tomography images," J. Nucl. Med., vol. 34, no. 11, pp. 2009-2018, 1993.

[68] P. A. Viola, "Alignment by maximization of mutual information," $\mathrm{Ph} . \mathrm{D}$. dissertation, Massachusetts Inst. Technol., Cambridge, MA, 1995.

[69] P. J. Slomka, P. Radau, G. A. Hurwitz, and D. Dey, "Automated threedimensional quantification of myocardial perfusion and brain SPECT," Comput. Med. Imag. Graph., no. 25, pp. 153-164, 2001.

[70] G. J. Klein, B. W. Reutter, and R. H. Huesman, "Four-dimensional affine registration models for respiratory-gated PET," IEEE Trans. Med. Imag., vol. 48, pp. 756-760, Mar. 2002

[71] T. G. Turkington, T. R. DeGrado, M. W. Hanson, and R. E. Coleman, "Alignment of dynamic cardiac PET images for correction of motion," IEEE Trans. Nucl. Sci., vol. 44, pp. 235-242, Apr. 1997.

[72] M. K. O'Connor, K. M. Kanal, M. W. Gebhard, and P. J. Rossman, "Comparison of four motion correction techniques in SPECT imaging of the heart: A cardiac phantom study," J. Nucl. Med., vol. 39, pp. 2027-2034, 1998.

[73] M. K. O'Connor, "Evaluation of motion-correction techniques in cardiac SPECT," J. Nucl. Med., vol. 41, no. 7, p. 1298, 2000.

[74] V. Bettinardi, M. C. Gilardi, G. Lucignani, C. Landoni, and G. Rizzo, "A procedure for patient repositioning and compensation for misalignment between transmission and emission data in PET heart studies," J. Nucl. Med., vol. 34, no. 1, pp. 137-142, 1993.

[75] C. M. Gallippi and G. E. Trahey, "Automatic image registration for MR and ultrasound cardiac images," in Lecture Notes in Computer Science 2082: Information Processing in Medical Imaging, IPMI01, M. F. Insana and R. M. Leahy, Eds., 2001, pp. 141-147.

[76] S. L. Bacharach, M. A. Douglas, R. E. Carson, P. J. Kalkowski, N. M. Freedman, P. Perrone, and R. O. Bonow, "Three-dimensional registration of cardiac positron emission tomography attenution scans," Comput. Vision, Graph. Image Process., vol. 34, no. 2, pp. 311-321, 1993.

[77] D. L. G. Hill and D. J. Hawkes, Handbook of Medical Imaging: Processing and Analysis. New York: Academic, 2000, ch. Acrossmodality registration using intensity-based cost functions, pp. 537-553.

[78] R. P. Woods, S. R. Cherry, and J. C. Mazziotta, "Rapid automated algorithm for aligning and reslicing PET images," J. Comput. Assist. Tomogr., vol. 17, no. 4, pp. 620-633, 1992.

[79] R. P. Woods, J. C. Mazziotta, and S. R. Cherry, "MRI-PET registration with automated algorithm," J. Comput. Assist. Tomogr., vol. 17, no. 4, pp. 536-546, 1993.

[80] N. Alpert, J. Bradshaw, D. Kennedy, and J. Correia, "The principal axes transformation-A method for image registration," J. Nucl. Med., vol. 31, no. 1 , pp. 717-721, 1990. 
[81] R. Kim, T. Aw, S. Bacharach, and R. Bonow, "Correlation of cardiac MRI and PET images using lung cavities as landmarks," in Proc. IEEE Conf. Computers in Cardiology, 1991, pp. 49-52.

[82] F. Maes, A. Collignon, D. Vandermeulen, G. Marchal, and P. Suetens, "Multimodality image registration by maximization of mutual information," IEEE Trans. Med. Imag., vol. 16, pp. 187-198, Apr. 1997.

[83] W. M. Wells, P. Viola, H. Atsumi, S. Nakajima, and R. Kikinis, "Multi-modal volume registration by maximization of mutual information," Med. Image Anal., vol. 1, no. 1, pp. 35-51, 1996.

[84] R. P. Woods, Handbook of Medical Imaging: Processing and Analysis. New York: Academic, 2000, ch. Within-modality registration using intensity-based cost functions, pp. 529-536.

[85] P. J. Slomka, D. Dey, C. Przetak, and R. Baum, "Automated 3-D spatial integration of 18-F FDG wholebody PET with CT," J. Nucl. Med., vol. 41 , no. 6, p. 59P, 2000.

[86] _ " "Automated nonlinear 3-D registration of 18-F FDG wholebody PET with thoracic CT," J. Nucl. Med., vol. 42, no. 5, p. 11P, 2001.

[87] J. Lötjönen and T. J. Mäkelä, "Elastic matching using a deformation sphere," in Lecture Notes in Computer Science 2208: Medical Image Computing and Computer-Assisted Intervention, MICCAI01, W. Niessen and M. Viergever, Eds., 2001, pp. 541-548.

[88] P. Thévenaz, T. Blu, and M. Unser, Handbook of Medical Imaging: Processing and Analysis. New York: Academic, 2000, ch. Image interpolation and resampling, pp. 393-418.

[89] M. Haddad, "Impact of reorientation algorithms on quantitative myocardial SPECT perfusion imaging," J. Nucl. Med., vol. 39, no. 11, pp. $1864-1869,1998$

[90] J. Nelder and R. A. Mead, "A simplex method for function minimization," Comp. J., vol. 7, pp. 308-313, 1965

[91] R. P. Woods, Handbook of Medical Imaging: Processing and Analysis. New York: Academic, 2000, ch. Validation of registration accuracy, pp. 491-497.

[92] —-, Handbook of Medical Imaging: Processing and Analysis. New York: Academic, 2000, ch. Spatial transformation models, pp. 465-490.

[93] M. Dahlbom and S.-C. Huang, Handbook of Medical Imaging: Processing and Analysis. New York: Academic, 2000, ch. Physical and biological bases of spatial distortions in positron emission tomography images, pp. 439-448.

[94] G. W. Goerres, E. Kamel, T.-N. H. Heidelber, M. R. Schwitter, C. Burger, and G. K. von Schulthess, "PET-CT image co-registration in the thorax: Influence of respiration," Eur. J. Nucl. Med., vol. 29, no. 3, pp. 351-360, 2002

[95] J. N. Yu, F. H. Fahey, H. D. Gage, C. G. Eades, and J. W. Keyes, "Evaluation of emission-transmission registration in thoracic PET," J. Nucl. Med., vol. 35, no. 11, pp. 1777-1781, 1994.
[96] W. L. Costa, D. R. Haynor, and T. K. Lewellen, "Registration of segmented attenuation and emission data in PET," in Proc. Nucl. Science Symp. Medical Imaging Conf., 1994, pp. 1407-1411.

[97] C. D. Stone, J. W. McCormick, D. R. Gilland, K. L. Greer, R. E. Coleman, and R. J. Jaszczak, "Effect of registration errors between transmission and emission scans on a SPECT system using sequential scanning," J. Nucl. Med., vol. 39, no. 2, pp. 365-373, 1998.

[98] S. W. Alderson, L. H. Lanzl, M. Rollins, and J. Spira, "An instrumented phantom system for analog computation of treatments plans," Amer. J. Roentgenol. Radium Ther. Nucl. Med., vol. 87, pp. 185-195, 1962.

[99] P. H. Pretorius, W. Xia, M. A. Kinag, B. M. W. Tsui, T. S. Pan, and B. J. Villegas, "Evaluation of right and left ventricular volume and ejection fraction using a mathematical cardiac torso phantom," J. Nucl. Med., vol. 38 , no. 10 , pp. $1528-1535,1997$.

[100] T. Beyer, D. W. Townsend, T. Brun, P. E. Kinahan, M. Charron, R. Roddy, and J. Jerin, "A combined PET/CT scanner for clinical oncology," J. Nucl. Med., vol. 41, no. 8, pp. 1369-1379, 1999

[101] J. Patton, D. Delbeke, and M. P. Sandler, "Image fusion using an integrated, dual-head coincidence camera with X-ray tube-based attenuation maps," J. Nucl. Med., vol. 41, no. 8, pp. 1364-1368, 2000.

[102] W. J. Rogers, E. P. Shapiro, J. L. Weiss, M. B. Buchalter, F. E. Rademakers, M. L. Weisfeldt, and E. A. Zerhouni, "Quantification of and correction for left ventricular systolic long-axis shortening by magnetic resonance tissue tagging and slice isolation," Circulation, vol. 84, no. 2 , pp. 721-731, 1991.

[103] W. G. O’Dell, C. C. Moore, W. C. Hunter, E. A. Zerhouni, and E. R. McViegh, "Three-dimensional myocardial defomations: Calculation with displacement field fitting to tagged MR images," Radiology, vol. 195, pp. 829-835, 1995.

[104] T. J. Mäkelä, Q. C. Pham, P. Clarysse, J. Lötjönen, K. L. H. Hänninen, J. Nenonen, J. Knuuti, T. Katila, and I. E. Magnin, "A 3-D model-based approach for the PET-functional and MR-anatomical cardiac imaging data fusion," in Lecture Notes in Computer Science 2230: 1st Int. Workshop Functional Imaging and Modeling of the Heart, FIMH 2001, T. Katila, I. E. Magnin, P. Clarysse, J. Montagnat, and J. Nenonen, Eds., 2001, pp. 83-90.

[105] C. Muller, M. Rombaut, and M. Janier, "Dempster shafer approach for high level data fusion applied to the assessment of myocardial viability," in Lecture Notes in Computer Science 2230: 1st Int. Workshop Functional Imaging and Modeling of the Heart, FIMH 2001, T. Katila, I. E. Magnin, P. Clarysse, J. Montagnat, and J. Nenonen, Eds., 2001, pp. 104-112.

[106] T. McInerney and D. Terzopoulos, "Deformable models in medical image analysis: A survey," Med. Image Anal., vol. 1, no. 2, pp. 91-108, 1996. 